\title{
LAS MONEDAS DE NECESIDAD DEL ESTADO DE MICHOACAN
}

$\mathbf{P} O \mathbf{R}$

\section{MANUEL ROMERO DE TERREROS}

\begin{abstract}
$\lambda^{\text {los guitones que se enumeran en estas páginas no puede darse con propie- }}$ A dad el nombre de monedas obsidionales, por no haber sido batidas durante el sitio de una plaza, pero sí cabe aplicarles el casi sinónimo dictado de monedas de necesidad, porque fueron acuñadas para llenar una apremiante necesidad local.
\end{abstract}

En diversas ocasiones y en varias comarcas de México (como en otros paises), la falta de moneda fraccionaria, indispensable para las operaciones comerciales de poca cuantía, y muy especialmente para las "rayas" en las haciendas, hizo que se buscara el remedio y, en consecuencia, se ideó la emisión de signos de cambio de valores representativos.

Antes y después de la Independencia, conociëronse estas fichas con el nombre de tlacos, y representaban generalmente la octava parte de un real, osea, la mitad de una cuartilla. Circularon en diversos puntos del país y se fabricaron no sólo de cobre, sino también de plomo, latón, madera, cuero, vidrio y hasta jabón; pero en este ensayo se consideran exclusivamente las del primer metal y sus aleaciones, emitidas y puestas en circulación en el Estado de Michoacán durante buena parte del siglo XIX, por constituir dichas piezas la serie más homogénea y numerosa de esta especie numismática. La tan 
extensa como importante Entidad Ferlerativa no acuño monedas oficiales propias, como to hicieron Jalisco, Zacatecas, Chihuahua y otrus Estados, en donde hubo plétora de moneda fraccionaria para satisfacer las necesidades regionales y prediales.

Los guitones de Michoacán no se llamaron solamente tlacos; algunos se designaron tantcos, arbitrios públicos y responsabilidades, nombres todos, sin embargo, que tenían prácticamente el mismo significado.

Además de estas monedas creadas por los Municipios, hay que tener en cuenta las que corrian en ciertas circunscripciones rurales, como ranchos y haciendas, en donde la población era a veces tan numerosa como la de una villa. También deben contarse entre las municipales las monedas de negociaciones particulares que llevan el nombre del Municipio en que se hallaban ubicadas, puesto que la circulación monetaria de los poblados cortos dependía muchas veces de un reducido número de casas de comercio. Por otra parte, el apellido que ostentan algunas monedas lo mismo puede haber sido de un funcionario municipal que de un comerciante.

Las necesidades públicas obligaban a ciertas poblaciones como Jiquilpan, Sahuayo y Huarachita, a formar pequeñas ligas monetarias y la circulación de su moneda no pasaba de su Municipio, a menos de que fuera sellada por otras comarcas con sus sendos signos convencionales.

Muchos pueblos de Michoacán, limitrofes del Esstado de Jalisco, usaban la moneda de éste (generalmente cuartillas muy gastadas) reselladas con iniciales o inscripciones abreviadas.

Ell Distrito que tuvo mayor circulación de monedas de necesidad fué indudablemente el de Zamora, en donde Municipios y comerciantes emitieron cantidad de fichas de esta especie, con diversos emblemas, apellidos y monogramas que hoy en dia es casi imposible identificar.

Por causas que se ignoran, pero seguramente por considerar dichas monedas ilegales el Gobierno del Estado o el de la Federación, las de Zamora fueron recogidas dos veces, pero otras tantas fueron puestas de nuevo en circulación; prueba de ello es que muchas llevan el resello $Z^{\text {a }}$ (Zamora) y no pocas, además, el de la fracción 1/8, a pesar de ostentar ya desde su origen el nombre del Municipio y el valor de la moneda.

En medio de los atributos puestos de moda en México desde la época de la Independencia, como son arcos y flechas, águilas y serpientes, nopales y gorros frigios, persistieron, sin embargo, en algunas monedas de Michoa- 
cán emblemas y adornos del tiempo de la Colonia. Tates son el busto de Carlos IV y el Escudo Real de España en las monedas de Cotija, y el collar de la Orden del Toisón de Oro en la de Turicuato. Peto si este hecho se explica por razones de tradición e historia, causa desagradable sorpresa encontrarse, también en una moneda de Cotija, una burda representación del águila y del escudo de las armas norteamericanas.

La mayoria de las monedas de Michoacán fué defectuosamente labrada a martillo desde un principio: en muchas leyendas hay faltas de ortografía y letras invertidas, los reversos no siempre corresponden en dirección a los anversos, y los tejos varían de grueso y no son siempre regulares. Pero estas monedas se encuentran todavía más deterioradas por el uso y por el tiempo. Sin embargo, los diseños que ostentan demuestran que, de haber contado los Municipios con buenos elementos, se hubieran producido ejemplares nunismáticos verdaderamente artísticos.

\section{MUNICIPIOS $Y$ HACIENDAS DE MICHOACAN EN DONDE SE EMITIERON} "MONEDAS DE NECESIDAD". *

ANGAMACUTIRO.-Pueblo. Municipalidad del Distrito de Puraándiro. APATZINGAN.-Villa. Municipalidad del Distrito de Tancitaro.

ARIO.-Villa, Municipalidad ; Distrito de su nombre.

BUENAVISTA.-Hacienda. Municipalidad de Ixtlán.

COCUPAO (QUIROGA).-Villa, Municipalidad y Distrito de su nombre.

COTIJA.-Pueblo. Municipalidad del Distrito de Jiquilpan.

CHARO.-Villa. Municipalidad y Distrito de Morelia.

CHAVINDA.-Pueblo. Municpialidad del Distrito de Zamora.

CHILCHOTA.-Pueblo. Municipalidad del Distrito de Tangancícuaro.

CHURINCIO.-Pueblo. Municipalidad de Penjamillo, Distrito de Purépero. quilpan.

GUARACHITA ó HUARACHITA.-Pueblo. Municipalidad del Distrito de Ji-

HUERTA.-Dos haciendas; una en la Municipalidad de Morelia y la otra en la de Tancítaro.

IXTLAN.-Pueblo. Municipalidad del Distrito de Zamora.

JIQUILPAN.-Pueblo. Municipalidad y Distrito de su nombre.

MORELIA:-Capital del Estado. Municipalidad y Distrito de su nombre.

NAHUATZEN.-Pueblo. Municipalidad del Distrito de Paracho.

* Como la división territorial del Estado de Michoacán ha variado en fechas más recientes, esta nómina se ha formado de acuerdo con las "Noticias". de D. José Guadalupe Romero, por ser su obra casi contemporánea de las monedas que se describen. 
PATZCUARO.-Ciudad. Municipalidad y Distrito de su nombre. PENJAMILLO.-Villa. Municipalidad del Distrito ¿e Purépero. LA PIEDAD.-Ciudad. Municipalidad y Distrito de su nombre. EL PILON.-Hacienda. Municipalidad del Distrito de Tancítaro. PUREPERO.-Villa. Municipalidad y Distrito de su nombre. PURUARAN.-Hacienda. Municipalidad del Distrito de Tacámbaro. LOS REYES.-Villa. Municipalidad del Distrito de su nombre. SAHUAYO.-Pueb!o. Municipalidad del Distrito de Jiquilpan. SAN RAFAEL.-Hacienda. Municipalidad de Turicato. Distrito de Tacámbaro. SANTA CLARA.-Villa. Municipalidad del Distrito de Pátzcuaro. SANTA EFIGENIA.-Hacienda. Municipalidad y Distrito de Ario. TACAMBARO.-Ciudad. Municipalidad y Distrito de su nombre. TANCITARO.--Villa. Municipaiidad del Distrito de su nombre.

TANGAMANDAPIO (SANTIAGO).-Pueblo. Municipalidad del Distrito de Zamora.

TANGANCICUARO-Villa. Municipalidad del Distrito de su nombre.

TANHUATO.-Pueblo. Municipalidad del Distrito de la Piedad.

TARETAN.-Villa. Municipalidad del Distrito de Uruapan.

EL TEJAMANIL.- Hacienda. Municipalidad y Distrito de Ario.

TINGUINDIN.-Pueblo. Municipalidad del Distrito de los Reyes.

TIPITARILLO.-Hacienda. Municipalidad del Distrito de Uruapan.

TIPITARO.-Hacienda. Municipalidad del Distrito de Uruapan.

TLAZASALCA.-Pueblo. Municipalidad del Distrito de Purépero.

TOMENDAN.-Hacienda. Municipalidad del Distrito de Uruapan.

TURICATO.-Villa. Municipalidad del Distrito de Tacámbaro.

URUAPAN.-Ciudad. Municipalidad del Distrito de su nombre.

ZAMORA.Ciudad. Municipalidad y Distrito de su nombre.

\section{ANGAMACUTIRO}

1 Anverso.-El águila mexicana. Leyenda: (1) ANGAMACUTIRo. 1855.

Reverso.-Un carcax con flechas. Leyenda: FONDO MUNICIPAL. UN OCTAVo.

Gráfilas de pequeñas rayas. Canto liso. (2)

Cobre. $24 \mathrm{~mm}$.

\section{APATZINGAN (3)}

2 Anverso.- Jarrón de flores colocado sobre un pedestal. Leyenda: TANTEO DE APATZINGAN.

(1) Leyendas son los letreros curvos; inscripciones, los horizontales.

(2) A menos que se exprese lo contrario, todas estas monedas tienen el canto liso.

(3) Lugar en donde Morelos, Licéaga, el Dr. Coss y otros insurgentes firmaron la primera Constitución Mexicana, el 24 de octubre de 1814. 
Reverso.-En el centro, monograma de las letras M. E., cursivas. Leyenda: UN OCTAVo. AÑo. En el exergo, 1847.

Gráfilas de figuras aspadas. Cordoncillo de impresiones rectangulares.

Latón. $20 \mathrm{~mm}$.

Moneda particular.

3 Igual a la anterior, pero del añs de 1850 y con gráfilas de adorno.

Los ejemplares que conocemos de estas monedas están contramarcados, al anverso. con un dos (2), incuso.

\section{ARIO}

4 Anverso.-Debajo de la inscripción ARIO, la fracción 1/8; a la izquierda un florón. Gráfila de picos.

Reverso.-Liso.

Cobre. $22 \mathrm{~mm}$.

5 Anverso.-Dentro de un círculo, el monograma de las letras M O A R I (Municipio de Ario). Gráfila de puntos.

Reverso.-Dentro de una corona de palmas, la fracción $1 / 8$.

Leyenda: PADILLA. A, D, 1826.

Cobre. $17 \mathrm{~mm}$.

6 Igual a la anterior, pero del año de 1830.

7 Anverso.-Leyenda: ARIO. La fracción 1/8 y abajo la fecha 1831, rodeadas de dos ramas de laurel.

Reverso.- - L iso.

Cobre. $22 \mathrm{~mm}$.

8 Anverso-En el centro, cuerno de la abundancia y otros adornos, como palmas y ramas de laurel. Leyenda: ARIO, 1843. Gráfilas de rayas. Todo burdamente ejecutado.

Reverso.-Liso.

Cobre. $23 \mathrm{~mm}$.

9 Anverso.--En el centro, cabeza para la derecha. Leyenda: MUNICIPALIDAD DE ARIO. En el exergo, la fecha 1858.

Reverso.-Dentro de una corona de laurel, la fracción $1 / 8$.

Gráfilas de pequeñas rayas.

Cobre. $20 \mathrm{~mm}$.

10 Anverso.-Figura que parece una fuente. Leyenda: MUNICIPALIDAD DE ARIO. 1865.

Reverso.--Dentro de una corona de laurel y encina, la fracción 1/8.

Gráfila de rayas.

Cobre. $23 \mathrm{~mm}$. 


\section{BUENAVISTA}

11 Anverso.-En el centro, las iniciales J. F. V. (José Francisco Velarde) (4) enlazadas. Leyenda: HACIENDA DE BUENAVISTA.

Reverso.-Dentro de un círculo con prolongaciones diametrales (probablemente el "fierro" de la finca), la fecha 1855. Leyenda: RACIONES DE MAIZ VALE POR 1 ALMUD.

Gráfilas de puntos.

Cobre. $28 \mathrm{~mm}$.

12 Anverso.-Dentro del fierro, la fecha 1855. Leyenda: HACIENDA DE BUENAVISTA. Reverso.-Las iniciales J. F. V., enlazadas.

Cobre. $22 \mathrm{~mm}$.

13 Anverso.-Las iniciales J. F. V., como en las anteriores.

Reverso.-Dentro del fierro de la finca, la fecha 1861. Leyenda: TIENDA DE BUENAVISTA.

Gráfilas de puntos.

Cobre. $24 \mathrm{~mm}$.

\section{COCUPAO (5)}

14 Anverso.-Gorro frigio radiante. Leyenda: COCUPAO.

Reverso.-La fracción $1 / 8$ entre una rama de laurel y otra de encina, enlazadas. Gráfilas de rayas.

Bronce. $20 \mathrm{~mm}$. Vaciada.

Fué hecha por el comerciante Manuel Fuentes.

15 Anverso.-Dentro de un tosco círculo de puntos, las letras del nombre MANUEL FUENTES, en monograma; debajo, DE, y en el exergo. Cocupao, en cursivas.

(4) Don José Francisco Velarde era también dueño de la cercana hacienda de La Barca, en el Estado de Jalisco, cuya moneda era como sigue: Anverso: En el centro y dentro del fierro (igual al de Buenavista), la fecha 1857 ; leyenda: J. F. VELARDE; en el exergo, una vaca echada; completan la circunferencia dos ramas de encina. Reverso: Parecido al anverso, pero con un cerdo en lugar de la vaca, y la leyenda: TIENDA. DE LA BARCA.-Cordoncillo labrado. Cobre. $24 \mathrm{~mm}$. "Velarde", dice D. Luis Pérez Verdía en su Historia de Jalisco, "era un hombre muy acaudalado que vivía en Guadalajara o en La Barca, en donde poseía fincas rústicas y urbanas de considerable valor; y por su riqueza que lucia con mal gusto y poca cultura, se le conocía con el apodo de "burro de oro". Desde la época de Santa Anna ostentaba el grado de General, fué partidario decidido del Imperio, tanto que para alojar a Maximiliano a su paso para Guadalajara, mandó alhajar la casa de su Hacienda de La Barca, adornándola con pinturas que ejecutaron los mejores pintores de aquella ciudad. Cuando los republicanos se acercaban a La Barca, él no se consideró seguro y marchó a Zamora, en donde se vió obligado por su grado de General, a mezclarse en las operaciones militares de la defensa. Después de la ocupación se escondió, pero como no se presentó en el plazo estipulado en un banđo militar, fué denunciado por un barbero, y a principios de febrero de 1867. fué pasado por las armas".

(5) Hoy lleva el nombre de QUIROGA, en honor del ilustre Obispo don Vasto. quien estableció alli la industria de las bateas de madera. 
Reverso.-Liso.

Cobre. $19 \mathrm{~mm}$. Vaciada.

Fué hecha por Manuel Fuentes en 1838.

16 Anverso.-. Una montaña accidentada (el Tzirate). Leyenda: MUNICIPALIDAD DE COCUPAO. En el exergo. AÑo DE 1848.

Gráfila de adorno.

Reverso.-En el centro, la fracción 1/8, debajo de dos manos asidas, entre una rama de laurel y otra de oliva, cruzadas en su extremidad inferior.

Bronce. $23 \mathrm{~mm}$.

Fué hecha para celebrar la reconciliación efectuada entre dos bandos enemigos que, por causas baladies, habian dividido a los habitantes de Cocupao. Fué costeada por el Municipio y la ejecutó un platero de Pátzcuaro, llamado Bejatanó, quien récibió por su trabajo cincuenta pesos.

\section{COTIJA}

17 Anverso.-En el centro, la fracción 1/8. Leyenda: CotiJa. Co... (Comencio?). 1825.

Gráfila de adorno.

Reverso.-Matrona con el gorro frigio sobre tra bara en la mano. Leyenda: D. JOSE NUÑEZ (sic).

Cobre $20 \mathrm{~mm}$.

18 Anverso.-El águila mexicana. Leyenda: TANTEO- DE COTIJA. UN 1/8. 1856. Reverso.-Gorro frigio radiante, rodeado de ocho puntos. Leyenda: JOSE NUÑEZ. Ramas de laurel y encina.

Gráfilas de rayas.

Cobre $21 \mathrm{~mm}$.

19 Anverso.-El águila americana (?), Leyenda: TANTEO DE COTIJA. UN OTAVO (sic) 1856 .

Reverso.--Como el de la anterior.

Gráfilas de rayas.

Cobre $20 \mathrm{~mm}$.

20 Anverso.-Busto de Carlos IV. Rey de España, a la derecha, de coleta y de traje de corte. Leyenda: TANTEO-DE COTIJA: en el exergo. $18611 / 8 \ldots$

Reverso.-Escudo coronado de las Armas Reales de España, rodeado del Toisón.

Leyenda: -BARGAS - I-COMPAÑIA.

Gráfilas de pequeñas rayas. Cordón de líneas oblicuas parałelas.

Latón y cobre. $20 \mathrm{~mm}$. Hay ejemplares sobredorados.

Ofrece de curioso este tanteo el haber sido formado con el busto y emblema de una moneda de oro de Carlos IV. Quizás se aprovechase algún cuño colonial de desecho.

21 Anverso-Como el de la moneda anterior.

Reverso.-El águi'a mexicana. Leỳenda: $\rightarrow$ BARGAS- I-COMPAÑIA. Debajo de la leyenda, puntos de adorno. 
Gráfilas de pequeñas rayas.

Cobre. $22 \mathrm{~mm}$.

\section{CHARO}

22 Anverso.-Leyenda: CHARO. Completan el circulo once estrellas: en el centro, la fracción $1 / 8$.

Reverso.-Liso.

Cobre. $22 \mathrm{~mm}$.

\section{CHAVINDA}

23 Anverso.-Figura de mujer sentada, con un caduceo en la mano izquierda. Leyenda: CHABA.- 1848 .

Reverso.-Aguila mexicana. Leyenda: COM. DE R. TAM. (Comercio de Rafael Tamayo).

Gráfilas de adorno.

Cobre. $20 \mathrm{~mm}$.

24 Anverso.-Figura con traje talar, que representa una mujer de la comarca; el brazo izquierdo levanta en alto una varilla con el gorro frigio. Leyenda: CHABINDA. AÑo DE 1858.

Reverso.-El águila mexicana sobre el nopal. Leyenda: RAFAEL TAMALLO (sic). Gráfilas de pequeñas rayas.

Cobre. $23 \mathrm{~mm}$.

\section{CHILCHOTA}

25 Anverso.--El águila mexicana. Leyenda: CHILchora. En el exergo, la fecha 1854. Reverso.-Corona de laurel: en el centro la fracción 1/8. Leyenda: dentro de la corona: GOMEZ.

Cobre. $22 \mathrm{~mm}$.

26 Anverso.-Busto humano, desnudo, lauteado, de perfil para la derecha. Leyenda: CHILCHOTA VN OCTAVO. 1858.

Reverso.-Una rama florifera en el centro. Leyenda: RESPONSAVILIDAD (sic) DE MVRGVIA.

Cobre. $20 \mathrm{~mm}$.

27 Anverso.-.En el centro, la fracción 1/8. Leyenda: CHILCHOTA.

Reverso.-Un arco que cruzan dos flechas en sotuer. Leyenda: MORFIN.

Cobre. $22 \mathrm{~mm}$.

\section{CHURINCIO}

28 Anverso.-El águila mexicana. Leyenda: CHURINCIO.

Reverso.-Dentro de una corona de laurel, la fracción 1/8. En el exergo, la fecha 1851 . 
Gráfilas de pequeñas rayas.

Cobre. $20 \mathrm{~mm}$.

29 Anverso.-El äguila mexieana. Leyenda: ANTONIO AIVRZ.

Gráfilas de pequeñas rayas.

Reverso,-Dentro de un guirnalda de laurel, la fracción 1/8. Leyenda: CHURINCIO. Gráfila de puntos.

Cobre, $20 \mathrm{~mm}$.

\section{HUARACHITA}

30 En una cuartiila de Jalisco, (6) un sobresello con las letras M. D. H. (Municipio de Huarachita), dentro de un marco rectangular con adornos.

\section{HUER'TA}

31 Anverso.-Leyenda: hVERTa. Completa el círculo una guirnalda de laurel. En el centro, una roseta.

Reverso.-La fracción $1 / 8$, pero con un punto grueso en vez del numerador.

Cobre. $18 \mathrm{~mm}$.

32 Anverso.-Dentro de una corona foliácea, la inscripción en cinco líneas: 2. R. ACIENDA (sic) -DE LA - HUERTA.

AN. (sic) D. 1830.

Reverso.-Dentro de igual corona, el fierro de la hacienda, entre dos tamas de encina.

Cobre. $28 \mathrm{~mm}$.

Dos reales, o Peseta.

\section{IXTLAN (7)}

33 Anverso.-Leyenda: tamallo (sic) IZTLAN.

Reverso.-Dentro de una corona de laurel circulo de puntos y rayas.

Cobre. $21 \mathrm{~mm}$.

\section{JIQUILPAN, SAHUAYO, HUARACHITA}

34 Cuattilla de Jalisco, resellada al reverso con una marca elíptica de gráfilas de pequeñas rayas, con las letras J. S. G.-(Jiquilpan, Sahuayo, Guarachita) y, debajo. un corchete horizontal.

35 Como la anterior, pero con un segundo sello, también eliptico, con la letra F.

(6) La cuartilla de Jalisco era: Anverso.- La Libertad, sentada, y teniendo en la mano derecha, sobre una vara. el gorro frigio; leyenda: UN OCTAVO. Reverso.-Un arco y un carcax en sotuer, debajo de un pendón; leyenda: ESTADO DE JALISCO, y la fecha. Cobre. $31 \mathrm{~mm}$.

(7) Llamado también IXTLAN DE LOS HERVORES, debido a los muchos manantiales de aguas termales que alli hay. El P. Landívar, en su "Rusticatio Mexicana". describe "con admiración el fenómeno de una de esas fuentes, que suspende sa ebullición en el momento que se acerca a ella alguna persona". (Romero). 


\section{MORELIA}

36 Anverso.-Amorcilio para la izquierda, teniendo en la mano detecha el gorro frigio radiante en una vara: (8) guirnalda de laurel y encina.

Reverso.-Leyenda: MUNICIPALIDAD DE MORELIX. 1837. En el centro, la fracción $1 / 8$.

Gráfilas de pequeños picos.

Cobre. $28 \mathrm{~mm}$.

37 Anverso.-El águila mexicana. Leyenda: AÑo DE 1843.- 23 DE LA INDEPENDa Reverso.-En el centro, la fracción 1/8. Leyenda: VALGA P\$ LA MUNICIPALIDAD DE MORELIA. Gráfilas de pequeños picos. Cobre. $28 \mathrm{~mm}$.

38 Cuartilla de Jalisco, sellada en Los Reyes (NO 56) y resellada con las letras MA. dentro de matco a manera de cartouche.

\section{NAHUATZEN}

39 Anverso.- Un indio para la izquietda con una hacha al hombro, enter una palma y una rama de laurel. Leyenda: NAHUATZEN.

Reverso.-El águila mexicana. Leyenda: MOLINA.

Cobre y latón. $21 \mathrm{~mm}$.

40 Anverso.-Dentro de una corona de laurel, cerro cubierto de vegetación. Leyenda: MUNICTPIO DE NAHUATZEN.

Reverso.-Dentro de igual corona de laurel, busto de mujer para la izquierda. Leyenda: NARSISO (sic) RAMIREZ. En el exergo: 89 (?).

Cobre y latón. $22 \mathrm{~mm}$.

41 Anverso.-Dentro de un circulo de puntos, unas balanzas de platillo, entre las iniciales F. A. cursivas; debajo, la fracción 1/8. Leyenda: TANTEO MERCANTIL. NAHUATZEN.

Reverso.-Liso.

Cobre. $30 \mathrm{~mm}$.

\section{PATZCUARO}

42 Anverso.--El águila mexicana. Leyenda: MUNICIPD. DE PAZCUARO.

Reverso.-Dentro de una corona formada por una palma y una rama de encina, atadas por la extremidad inferior, la inscripción en dos lineas: 1/8. - 1856.

Cordoncillo de impresiones rectangulares.

Cobre. $32 \mathrm{~mm}$.

43 Anverso.-Las islas y aguas del lago; un indio en una canoa, rodeada de patos. Leyenda: MUNICIPD. DE PAZTCUARO (sie); debajo, la fraeción 1/8.

Reverso.-Una india de perfil que camina para la derecha, llevando en la mano unos pescados y cargado a la espalda un quimit (envoltorio de ropa).

Gráfilas de estrellas.

Cordoncillo de rayas verticales muy juntas.

Latón $x$ cobre. $23 \mathrm{~mm}$. catecas.

(8) Imitación del "Getio de la Libertad", en el anverso de las crortillas de Za- 
44 Anverso.- Montaña toscamente representada.

Gráfila de puntos.

Reverso--En el centro, la fracción 1/8. Lependa: PAZCUARO.

Gráfila de pequeños picos.

Cobre. $20 \mathrm{~mm}$.

45 Igual a la anterior, peto con la fracción y la leyenda más pequeñas.

Cobre. $18 \mathrm{~mm}$.

46 Igaal a la número 44 , pero con el sobresello: VALE, al anverso.

\section{PENJAMILLO}

47 Anverso.-Aguila mexicana. Leyenda: ARVITRIO (sic) PUBLICO.

Reverso.-El gorro frigio, tadiante. Leyenda: inferior: 1/8 PENJAMO. 1852. (Penjamillo).

Gráfilas de estrellas.

Latớn y. cobre. $22 \mathrm{~mm}$.

48 Tanteo mercantil de Nahuatzen (No 41). contramarcado al anverso con Po. (Penjamillo).

\section{PIEDAD}

49 En una cuartilla de la Federación, (9) o de Jalisco, muy lisa, la inscripción en dos lineas: PIEDAD - 1871, al anverso.

La hay también en tejos muy delgados; algunos con iriciales no identificadas.

\section{PILON}

50 Anverso.-Dentro de una guirnalda de laurel una montafla (EL PILON), detrás de la cual se pone el sol. Leyenda: TANTEO DEL PILON. En el exergo: *UN OCTAvo*. Reverso.-Dentro de igual guirnalda de laurel, dos A A cursivas, ligadas por una curva superior. Leyenda: *CONSTANCIA AL TRABAJO 1861."

Cobre. $20 \mathrm{~mm}$.

\section{PUREPERO}

51 Anverso.-Gorro de la Libertad, rodeado de rayos. Leyenda: MUNICIPALIDAD.

Reverso.-Serranía en medio de las aguas. Leyenda: DE PUREPERO, UN OCTAvo; debajo, la fracción 1/8. En el exergo, la fecha 1851.

Gráfilas de estrellas.

Cobre. $23 \mathrm{~mm}$.

52 Igual a la anterior, pero con gráfilas flordelisadas.

Latón. $23 \mathrm{~mm}$.

(9) Anverso:-El ágaila wobe el sopal. Leyenda: REPUBLICA MEXICANA. Reverso.-Dentro de una coroma de barzh la fracción 1/4; debajo, la levenda: M. A. 1843. $29 \mathrm{~mm}$. 


\section{PURUARAN}

53 Moneda de Moretia (No 37), resellada al anverso con un punzón circular, de 14 mm., que tiene, dentro de una corona de laurel, la leyenda: PURUARAN -CAULOTE; en el centro, y debajo la fracción $1 / 2$.

Medio real.

\section{REYES}

54 Anverso.-El águila mexicana. Leyenda: REYEZ (sic) 1842.

Reverso.-Figura de hombre caminando hacia la derecha; lleva en la mano un objeto redondo. Leyenda: AGAPITO ROJAS*UN OCTAVO.

Cordoncillo de líneas oblicuas paralelas.

Cobre. $20 \mathrm{~mm}$.

55 Ancerso.-El águila mexicana bajo gorro frigio radiante. Leyenda: TANTEO DE - LOS REYES.

Reverso.-Corona de olivo y encina. En el centro, la fracción $1 / 8$, rodeada de la leyenda: *RAMON TORRES - Y COMPA*.

Gráfilas de pequeñas rayas.

Latón y cobre. $21 \mathrm{~mm}$.

56 En una cuartilla de Jalisco, un sello eliptico muy prolongado, con gráfilas de rayas y las letras *R. S.* inicial y terminal del nombre de Los Reyes.

57 En un tlaco de la Federación. (10) el sello anterior. Al reverso, impresa de realce, la letra D.

58 El sobresello anterior en una cuartilla de Jalisco, contramarcada por el Municipio de Huarachita (No 30). A1 reverso, las letras Po. de Penjamillo.

59 El mismo sel'o al anverso de un tanteo metcantil de Nahuatzen.

\section{SAHUAYO}

60 Anverso.-Un coyote, vuelto para la izquierda, en pie. Leyenda: ZahusYo.

Reverso.-Guirnalda de dos ramas de laurel. En el centro, las iniciales J. M. A. (José Maria Arregui) cursivas y separadas. Leyenda inferior: AÑo DE 1856.

Grafilas de puntos.

Latón. $22 \mathrm{~mm}$.

\section{SAN RAFAEL}

61 Anverso.-.En el centro, las iniciales Y. C., cursivas. Leyenda: HACIENDA DE SAN RAFAEL.

(10) Anverso.- - La Libertad, sentada, con el brazo derecho sobre el libro de !a Ley y la mano izquierda sobre una lanza; las haces romanas junto al asiento: leyenda: LIBERTAD. Reverso.- En el centro de una corona de laurel, la inseripción OCTAVO DE REAL: abajo, la fecha y la zeca $M$. 
Gráfila de pequeños picos.

Reverso.-Dentro de una goimalda de olivo y encina, la fracción 1/4. Leyenda: 1847.

Gráfila de pequeñass tayas.

Cobre. $24 \mathrm{~mm}$.

\section{SANTA CLARA (11)}

62 Anverso.-Aguila mexicana.

Reverso.-Leyenda: SANTA Clara. En el centro, la fracción 1/8. Abajo, palma. y rama de laurel.

Gráfilas de tayas.

Cobre. $22 \mathrm{~mm}$.

\section{SANTA EFIGENIA}

63 Anverso.-Bajo un sol radiante, figura que parece una serpiente. Leyenda: HACIENDA DE SANTA EFIGENIA.

Reverso.-La fracción 1/8, entre ramas de olivo y encina. Leyenda: A. 1840.

Cobre. $22 \mathrm{~mm}$.

64 Anverso.-Las letras Y. A. cursivas, sirviendo una flecha de atravesaño a la $A$. Leyenda: HACIENDA DE SA. EFIGENIA.

Reverso.-Balanza de platillo sostenida por una mano. Debajo del fiel, la fracción $1 / 4$ con grandes caracteres. En el exergo, 1846.*

Gráfilas de puntos. Cordoncillo de impresiones rectangulares.

Latón Y cobre. $24 \mathrm{~mm}$.

Cuartille.

\section{TACAMBARO}

65 Anverso.-En el centro, la fracción 1/8; abajo, la fecha 1848. Leyenda: TACAMBARO. Dos ramas de larrel.

Reverso.-Un caduceo, entre varios adornos.

Grifila de pequeñas rayas.

Cobre. $29 \mathrm{~mm}$.

66 Anverso.-Un peñón y un lago, toscamente ejecutados.

Reverso.-En el centro, la fracción 1/8. Dos palmas completan la leyenda inferior:

TACAMBARO.

Gráfilas de rayas.

Cobre. $21 \mathrm{~mm}$.

(11) Llamada también SANTA CLARA DEL COBRE, porque don Vasco de Quiroga asignó a sus vecinos el oficio de caldereros de cobre, quienes labraban toda clase de piezas de dicho metal, con bastante perfección. 
67 Anverso.-En el centro la fracción 1/8. Leyenda: TACAMBaro. Dos ramas de laurel. Grafilas de puntos.

Reverso.-Un peñón y un lago, toscamente ejecutados.

Gráfila de pequeñas rayas.

Cobre. $21 \mathrm{~mm}$.

\section{TANCITARO}

68 Anverso.-Un peñón. Leyenda: TANCitaro. Guirnalda de encina y laurel. En el exergo, la fecha 1864.

Gráfila de puntos.

Reverso.-Bajo un gorro frigio radiante, la fracción 1/8. Guirnalda de encina y de laurel. Leyenda: FONDO MUNICIPAL.

Gráfila de estrellas.

Cobre. $24 \mathrm{~mm}$.

\section{SANTIAGO TANGAMANDAPIO}

69 Anverso.-Leyenda: SANTI (Santiago), con letra eursiva y desigual.

Reverso.-Liso.

Cobre. $17 \mathrm{~mm}$. Tejo muy delgudo

\section{TANGANCICUARO}

70 Anverso.-El águila mexicana. Leyenda: MANUEL MURGIA (sic).

Reverso.-En el centro, la fracción 1/8. Leyenda: TANGansicuaro. * * * 1842 * * * Gráfilas de rayas.

Cobre. $21 \mathrm{~mm}$.

71 Anverso.-En el centro, la fracción 1/8. Leyenda: TANGANSICUARO; en el exergo, *** $1842 * * *$.

Reverso.-Monograma de las letras T. A. S. O. (Tangansicuaro) debajo de tres florones.

Gráfila de hojas de laurel.

Cobre. $21 \mathrm{~mm}$.

72 Anverso.-El águila mexicana. Leyenda: TANGANsicuaro.

Reverso.-Un niño con un objeto redondo en la mano (Batea?). Leyenda: PEDRo.

-JIMENEZ. 1842.

Gráfila de medias lunas.

Cobre. $20 \mathrm{~mm}$.

TANHUATO

73 Anverso.-El águila mexicana. Leyenda: MUNICIPIO DE TANHUATO. 
Reverso.-Dos ramas tupidas de laurel, abajo atadas y separadas arriba por el gorto de la Libertad, radiante. En el centro la fracción 1/8; debajo, la fecha 1871. Gráfilas de puntos.

Cobre. $25 \mathrm{~mm}$.

74 Anverso.-El águila mexicana. Leyenda: MUNICIPIO DE TANHUATO.

Reverso.-Dentro de una guirnalda de laurel, interrumpida en la parte superior por un gorro frigio radiante, un chivo erguido sobre los cuartos traseros, para la izquierda, pero vuelta la cabeza para la derecha. En el exergo, *1871*.

Cobre. $24 \mathrm{~mm}$.

\section{TARETAN}

75 Anverso.-Leyenda: TARETAN; debajo, la inscripción en dos líneas: 1837.1/8; una palma y una rama de encina.

Reverso.-Liso.

Cobre. $22 \mathrm{~mm}$.

76 Anverso.-Guirnalda de laurel. Leyenda: TARETAN. Un florón. la fecha 1842, y debajo, la fracción $1 / 8$.

Gráfila de puntos.

Reverso.-Liso.

Cobre. $24 \mathrm{~mm}$.

77 Anverso.-Gorro frigio radiante. Leyenda: TARETAN, UN OCTAvo. En el exergo, $184 \ldots$

Reverso.-Una palma de izote, (12) naciendo entre dos peñas. Leyenda: FONDO MUNICIPAL.

Cobre. $20 \mathrm{~mm}$.

78 Anverso.-Igual al del número 75.

Reverso-- Liso, pero con un sobresello rectangular con la inscripción en tres lineas: RECUÑA -DOS- EN 1849.

Cobre. $22 \mathrm{~mm}$.

79 Anverso.-Una iglesia con un árbol a la derecha; debajo, la fracción $1 / 8$ entre dos florones. Leyenda: FONDO PUBLICO DE TARETAN.

Gráfila de picos.

Reverso.-Liso.

Cobre. $22 \mathrm{~mm}$.

80 La misma moneda que la anterior. pero resellada al reverso con la inscripción del número 78.

81 Anverso.-Cabeza de indio para la izquierda. Leyenda: TARETAN. UN OCTAVo. En exergo, 1856.

Gráfila de rayas.

(12) Palma que produce flores olorosas y blancas y un fruto parecido al banana; este no se aprovechaba, pero de aquéllas los españoles hacian dulce. De las hojas los indigenas fabricaban esteras y sacaban hilo para sus manufacturas. 
Reverso.-Una palma de izote, naciendo entre dos peñas. Leyenda: FONDO MUNICIPAL.

Gráfila de adorno.

Cobre. $20 \mathrm{~mm}$.

82 Igual a la anterior, pero del año de 1858. Latón.

83 Anverso.-Gorro frigio radiante. Leyenda: MUNICIPALIDAD DE TARETAN.

Reverso.--Una palma de izote, naciendo entre dos peñas. Leyenda: UN OCTAvo. Cobre. $23 \mathrm{~mm}$.

84 Anverso.-Leyenda: P. TARETAN. En el centro la fractión 1/8.

Reverso.-Liso.

Cobre. $22 \mathrm{~mm}$.

\section{TEJAMANIL}

85 Anverso.-En el centro, dentro de una corona de laurel, la fracción 1/8; Leyenda: HDA. DEL TEJAMANIL.

Reverso.-Dentro de otra corona de laurel, la fracción 1/8. Abajo, 1858.

Cobre. $19 \mathrm{~mm}$.

Todos los ejemplares están resellados con un punzón, incuso en forma de roseta.

86 Anverso.-Un volcán con una media luna a la izquierda. Leyenda: HDA. DEL. TEJAMANIL.

Reverso.-En el centro, una palma. Leyenda exergo, la fecha 1858 .

Cobre. $19 \mathrm{~mm}$.

Medio real.

\section{TINGUINDIN}

87 Cuartilla de Jalisco, resellada con las iniciales M. D. T. (Municipio de Tingüindín). al anverso. Al reverso, con un sello éíptico, dentro del cual está la fracción $1 / 8$ con apéndices en los extremos de la raya.

88 Tlaco de la Federación, con las letras T. N. en un sello elíptico con gráfilas de pequeños picos.

89 Cuartilla de Jalisco, sellada y resellada, respectivamente, con las letras T. N. (Tingüindín) y M. D. T. en dos punzones elípticos.

90 Cuartilla de Jalisco, con dos sobresellos elípticos: el primero, con el monograma de las letras F. M. cursivas; y el segundo, con las letras T. N.

91 Cuartilla de Jalisco, sellada en Jiquilpan, Sahuayo y Huarachita, (No 34) y contramarcada con las letras $T$ in, dentro de un sello eliptico.

92 Cuartilla de Jalisco, resellada en Los Reyes (N9 56) y contramarcada con las letras $M . D . T$. dentro de un rectángulo. 

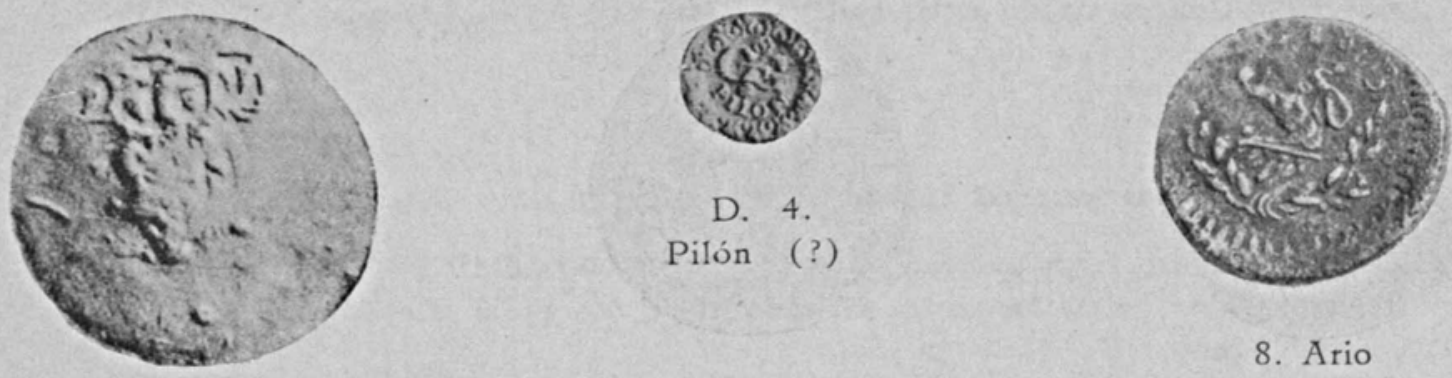

8. Ario

D. 1. Penjamillo

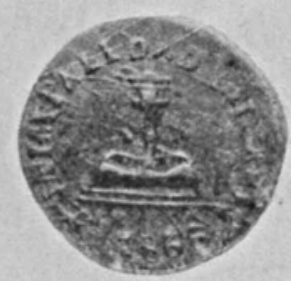

10. Ario

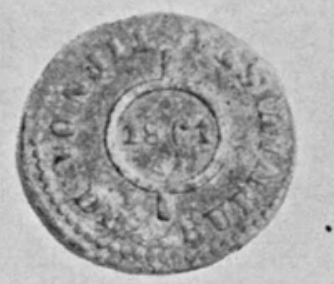

13. Buenavista

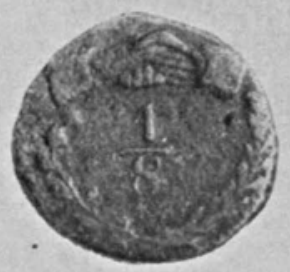

16. Cocupao

13. Buenavis

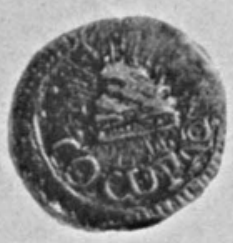

14. Cocupao

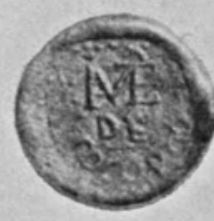

15. Cocupao

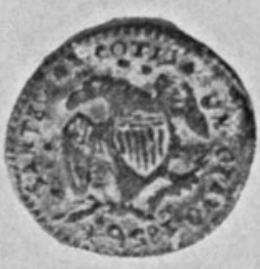

19. Cotija

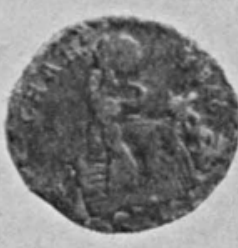

20. Cotija

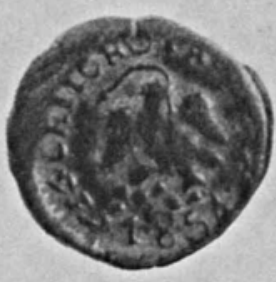

25. Chilchota

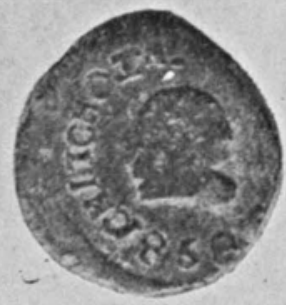

26. Chilchota 


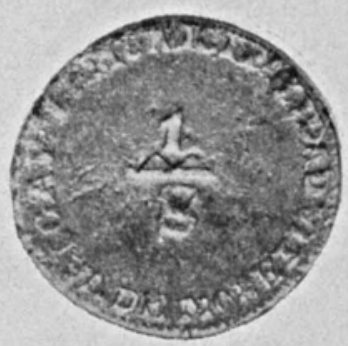

37. Morelia

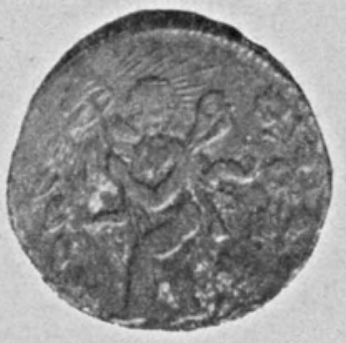

36. Morelia

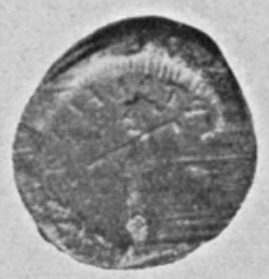

39. Nahuatzen
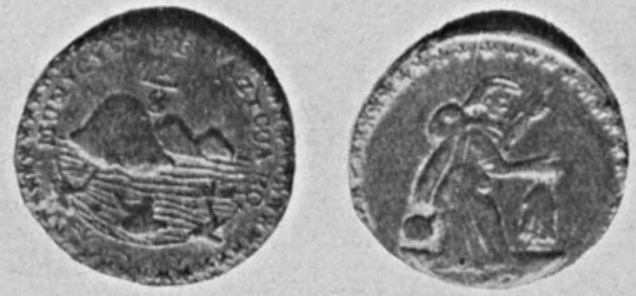

43. Pátzcuaro

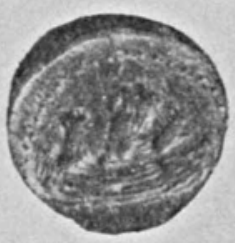

44. Pátzcuaro

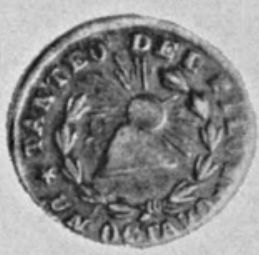

50. Pilón

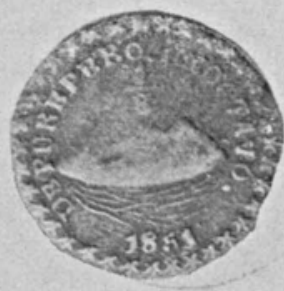

51. Purépero

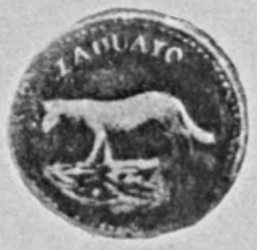

60. Sahuayo
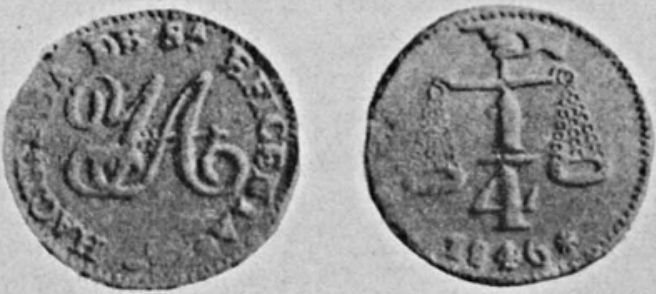

64. Santa Efigenia

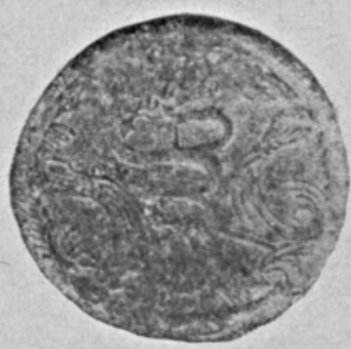

65. Tacámbaro 


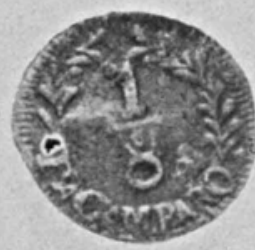

66. Tacámbaro

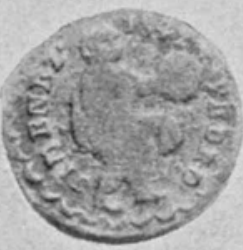

72. Tangancícuaro

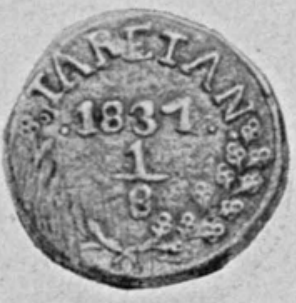

75. Taretan

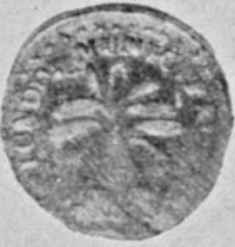

77. Taretan

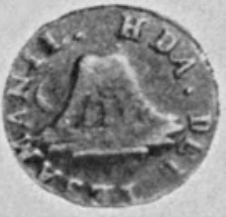

86. Tejamanil

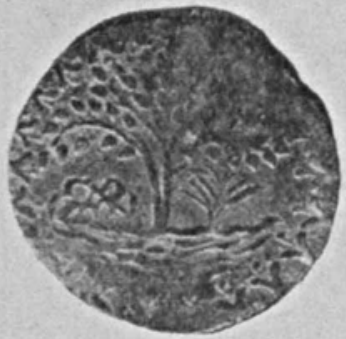

94. Tipitarillo

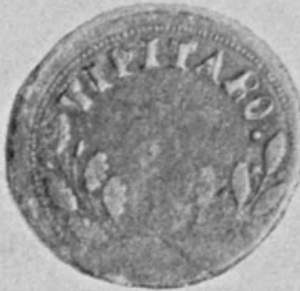

96. Tipitaro.

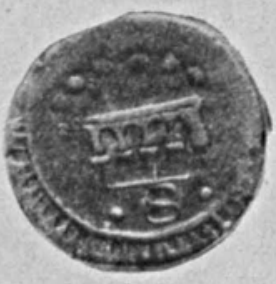

97. Tipitaro
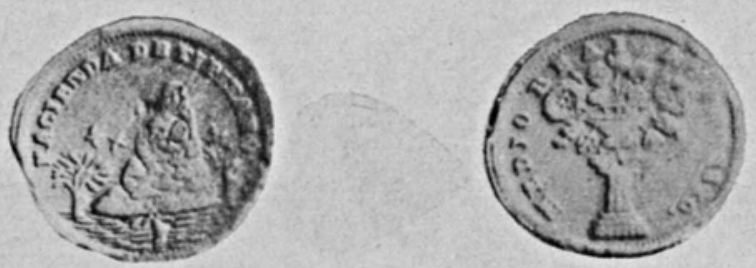

100. Tipitaro

(Anverso)

(Reverso) 


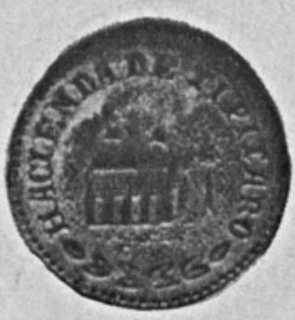

101. Tipítaro

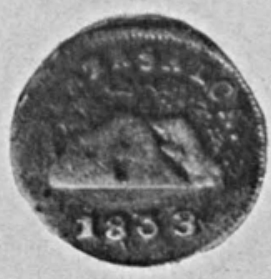

102. Tlazasalca

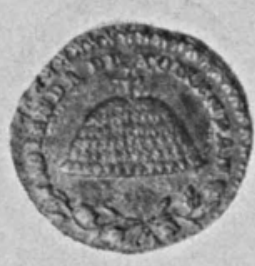

103 Tomendán

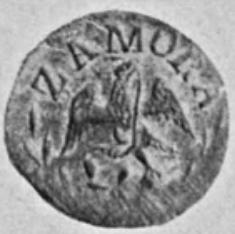

122. Zamora

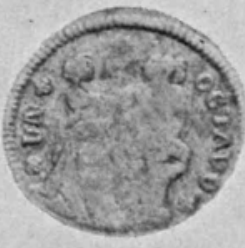

123. Zamora

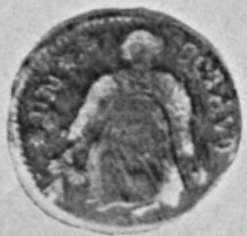

126. Zamora

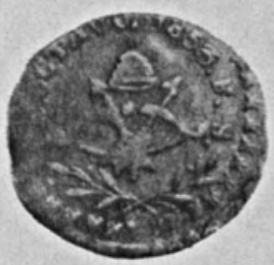

129. Zamora

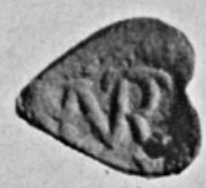

135. Zamora

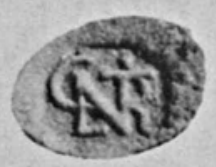

135. Zamora 


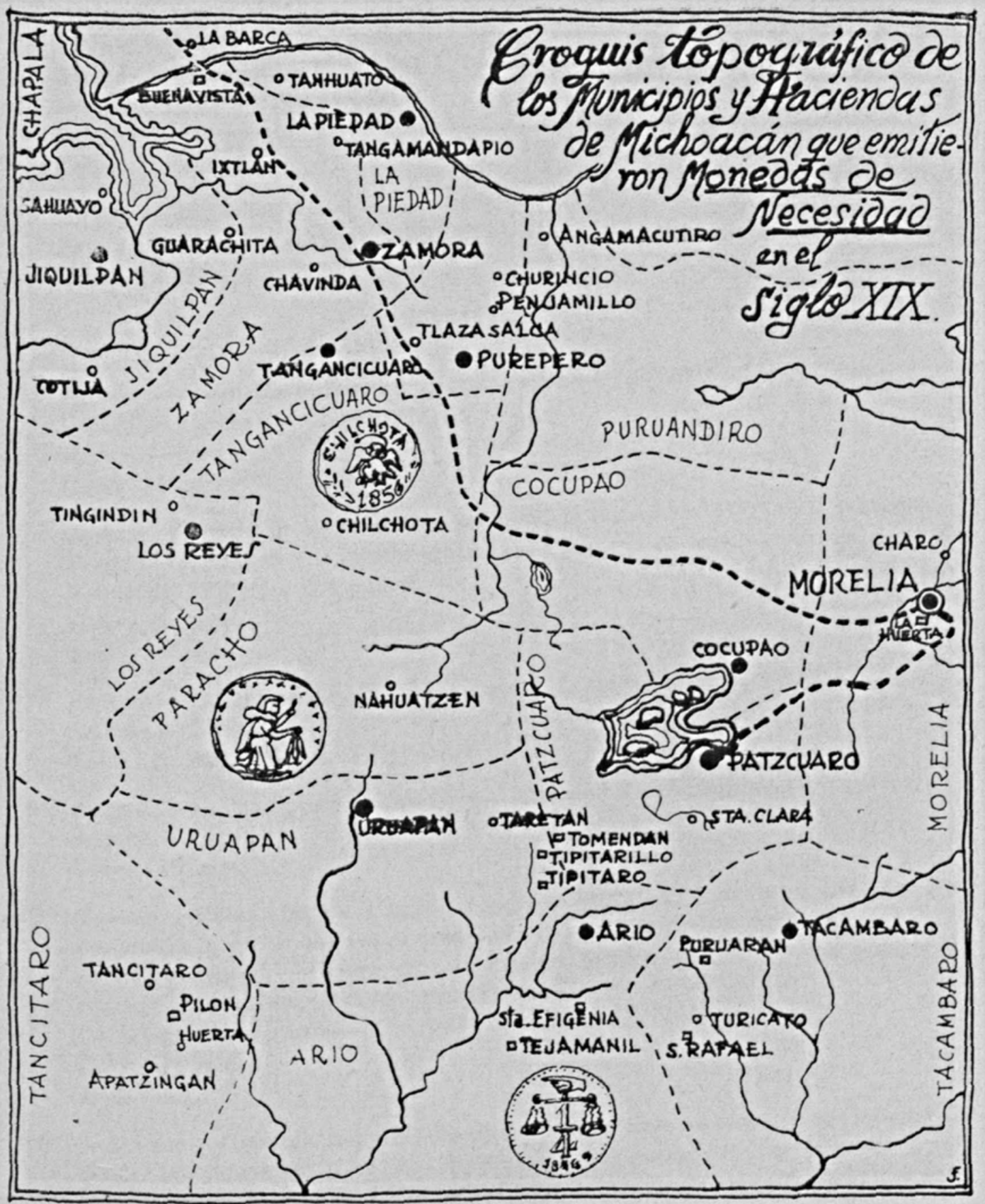


DOI: http://dx.doi.org/10.22201/iie.18703062e.1940.5.188 
93 En un tonteo de Taretan, la inscripción en dos líneas: M. D. T. - 1849, dentro de un óvalo con gráfila de puntos.

\section{TIPITARILLO}

94 Anverso.-Un árbol y dos arbustos.

Reverso.--En el centro, la fracción 1/8, rodeada de una palma y una rama de lapod.

Leyenda: 1850 -TIPITARILLO.

Gráfilas de adorno muy historiadas.

Cobte. $28 \mathrm{~mm}$.

95 Anverso.-Un árbol. En el exergo, la fecba 1855.

Reverso.--Un gallo. Leyenda: TIPITARILLO.

Cobre. $27 \mathrm{~mm}$.

\section{TIPITARO}

96 Anverso.-Guirnalda de laurel y encina. Leyenda: TIPITARo.

Gráfila de puntos.

Reverso.-Liso.

Cobre. $25 \mathrm{~mm}$.

97 Anverso.--Dentro de butda corona de laurel, la inscripción TIPITARO, subrayada Reverso.-Debajo de seis puntos gruesos, una arquería de cuatro arcos; en el exergo. la fracción $1 / 8$ entre dos puntos.

Gráfila de rayas.

Cobre. $22 \mathrm{~mm}$.

98 Anverso.-En el centro Mo (Municipio). Leyenda inferior: TPPftaro.

Reverso.--Liso.

Cobre. $26 \mathrm{~mm}$.

99 Anverso.-La inscripción en dos líneas: Mo. -TR. (Municipio de Tipitaro).

Reverso.--Liso.

Cobre, $20 \mathrm{~mm}$.

100 Anverso.-Peñón abrupto y tajado a pico, en medio del agua; a sa alrededor algunos árboles, y por el aire varias aves. Leyenda: HACIENDA DE TIPłTARO.

Reverso.-Pedestal en forma de columna que sostiene una maceta con plantas floríferas. Leyenda: MEDIO REAL. AÑo DE 1846.

Gráfilas de líneas gruesas.

Cobre. $24 \mathrm{~mm}$.

Medio real.

101.-Anverso.-Casa con techo de dos aguas; a sus lados un árbol p un arbusto. Leyenda: HACIENDA DE TIPITARO. En el exergo una guirnalda.

Reverso.-Dentro de una corona de laurel, la inscripción en dos renglones: UN REAL. Leyenda: DESE EN EFECTOS - FLORES-. 
$\because$ Gráfilas de puntos.

Cobre, $24 \mathrm{~mm}$.

Real.

\section{TLAZASALCA}

102 Anverso.-Cerro cubierto de vegetación. Leyenda; TLAZASALCA; en el exergo, la fecha 1853.

Reverso.-Dentro de una corona de laurel, la fracción $1 / 8$.

Cobre. $22 \mathrm{~mm}$.

\section{TOMENDAN}

103 Anverso.-Túmulo con una cruz en la cima. Leyenda: HACIENDA DE TOMENDAN. Dos ramas de encina.

Reverso,--Balanza de platillo sostenida por una mano. Debajo del fiel, la fracción $1 / 2$, entre una estrella y una $R$.

Gráfilas de estrellas.

Cobre. $20 \mathrm{~mm}$.

Medio real.

\section{TURICATO}

104 Anverso.-El águila mexicana. Leyenda: MUNICIPALIDAD DE TURICATO.

Reverso.-Dentro de un círculo de puntos, rodeado del collar del Toisón, la fracción $1 / 8$, entre las letras $N$. $M$.

Cobre: $24 \mathrm{~mm}$.

\section{URUAPAN}

105 Anverso-Dentro de un círculo, la inscripción en cuatro lineas: 8 -VRUA PAN - 26 (1826).

Gráfila de picos.

Reverso.-Liso.

Cobre y bronce. $21 \mathrm{~mm}$.

106 Anverso.-Dentro de una corona de laurel, la inscripción en dos líneas: URUAPAN -AÑ (sic) D 1830. Abajo, un florón.

Reverso.-Liso.

Cobre. $22 \mathrm{~mm}$.

106 a.-Parecida a la anterior, del año de 1831.

107 Anverso.-En el centro, un arco y dos flechas. cruzadas. Leyenda: URUAPAN 1854. Reverso.-En el centro, la fracción 1/8. Leyenda: IGNACIO BUENROSTRO. Rese- 
llado con un pequeño óvalo en forma de guirnalda, dentro del cual hay ara hoja y una letra $B$.

Cobre. $23 \mathrm{~mm}$.

108 Anverso.-El águila mexicana. Leyenda: URUAPAN. En el exergo, la fecha 1848, entre dos ramas de laurel.

Reverso.-Liso.

Cobre. $21 \mathrm{~mm}$.

112 Iguales a la anterior, pero de los años 1849, 1850,1852, 1856.

\section{ZAMORA}

113 Anverso.-Monograma de Samora (sic).

Reverso.-Dentro de un cuadro de muchos picos, la inscripción en dos líneas $\gamma$ en letras cursivas: CUIN (?) - 1800.

Cobre. $22 \mathrm{~mm}$.

Moneda urbana del Distrito de Zamora.

114 Anverso.-Dentro de una corona de laurel, la inscripción en cuatro líneas: BAA (Barrera?) 1 -OCTAvO- A. DE - 1837.

Reverso.-Dentro de dos círculos concéntricos, el exterior de adorno, un cuadrilátero con la fracción $1 / 8$.

Cobre. $20 \mathrm{~mm}$.

Moneda predial del Distrito de Zamora.

115 Anverso.-Dentro de una corona de laurel, la inscripción en cuatro líneas: CISs. (Cisneros) 1 - OCTAvo - A. DE - 1837.

Reverso.-Como el de la pieza anterior.

Cobre. $20 \mathrm{~mm}$.

116 Anverso.-Dentro de una corona de laurel, la inscripción en cuatto líneas: CIS (Cisneros) 1 OCTABO (sic) - $\mathrm{D} . \mathrm{DE}-1839$.

Reverso.-Como el de la pieza anterior.

Cobre. $20 \mathrm{~mm}$.

117 Anverso.-La inscripción en cuatro lineas: MAn. (Marrón) OCTABO (sic), -A. $\mathrm{DE}-1839$.

Reverso.-Doble gráfila de líneas.

Cobre. $20 \mathrm{~mm}$.

118 Anvetso.-Dentro de dos circulos concéntricos, el monograma de las letras F R N A D E (Fernández?).

Reverso.-Monograma de las letras CUAR (?); dentro de la C, la fecha 1839. Cobre. $18 \mathrm{~mm}$.

Moneda predial.

119 Anverso.-Dentro de una orla de ramajes, monograma de las letras J A M P E (?). Reverso.-En el centro, la fracción 1/8. Leyenda: LIBERTAD. En el exergo, AÑo DE 1840 . 
Cobre. $18 \mathrm{~mm}$.

Moneda predial.

120 Anverso:-El águila mexicana. Leyenda: ZAMORA, AÑO DE...

Reverso.-Dentro de un círculo de adorno, el monograma de las letras J A M P. cursivas.

Cobre. $15 \mathrm{~mm}$.

121 Anverso.-Dentro de un círculo, el águila mexicana. Leyenda: ZAMORA. AÑo DE... Reverso.-Monograma de las letras cursivas A M P.

Gráfila de adorno.

Cobre. $19 \mathrm{~mm}$.

122 Anverso.-El águila mexicana. Leyenda: ZAMORA.

Reverso.-Leyenda: J. M. GARCIA; en el resto del contorno dos ramas foliáceas. En el centro, la inscripción en dos líneas $1840-1 / 8$.

Latón y cobre. $20 \mathrm{~mm}$.

Moneda urbana de negociación mercantit.

123 Anverso.-Aguila mexicana. Leyenda: ZAMORA. 1842.

Reverso.-Matrona sentada en pedestal, hacia la izquierda, con el gorro frigio de la Libertad sostenido en una varilla en la mano derecha, y con la izquierda descansando sobre la cornucopia. (13) Leyenda: *UN*- OCTAvo*.

Cordoncillo labrado con figuras.

Latón. $20 \mathrm{~mm}$.

Moneda municipal. Llamada por el vulgo "Tlaco de la mona".

124 Anvetso,-E1 águila mexicana. Leyenda: ZAMORA 1842.

Gráfila de rayas.

Reverso,-Matrona como en la anterior. Leyenda: *UN*-OCTABO *. (sic).

Cobre. $20 \mathrm{~mm}$.

125 Igual a la anterior, pero del año de 1846.

126 Anverso.-El águila mexicana. Leyenda: ZAMORA. 1848.

Reverso,-Matrona sentada de frente, pero con el rostro vuelto para la izquiesda y los brazos pendientes a los lados del cuerpo: la mano derecha empuña una rama y la izquierda una tela. Leyenda: UN —OCTAvo.

Cobre. $20 \mathrm{~mm}$.

Moneda municipai.

127 Anverso.-El águila mexicana dentro de un círculo. Leyenda: ZAMORA. AÑ DE 1850. Reverso.-Dentro de dos círculos concéntricos, el monograma de las letras $B$ F R D O.

Cobre. $18 \mathrm{~mm}$.

128 Anverso.-El águila mexicaña.

Reverso - Dentro de un círculo la fracción $1 / 8$, y debajo el año 1851 .

(13) Imitación del cuño de Jalisco. 
Cobre. Eliptica. 21 por $19 \mathrm{~mm}$.

Moneda predial.

129 Anverso.- El águila mexicana. Leyenda: MUNICIPIO DE ZAMORA.

Reverso.- Arco dispuesto en dirección horizontal y cruzado por dos flechas en sotuer, con las puntas hacia arriba; el gotro frigio entre las puntas de las flechas. Leyenda: UN OCTAvo. 1853. F. S.

Gráfilas de adorno.

Cordoncillo de impresiontes tectangulares.

Cobre $22 \mathrm{~mm}$.

Moneda municipal.

130 Igual a la anterior, pero, del año de 1854.

131 Anverso.-Arco con dos flechas cruzadas en sotuer. Leyenda: MORFIN - 1854. Reverso.-Dentro de una corona de ramajes, la fracción $1 / 8$.

Cobre. $21 \mathrm{~mm}$.

Moneda urbana mercantil.

132 Igual al numero 129 , pero del año de 1855.

133 Igual al número 129, pero del año de 1857 , y con A. L.

134 Igual al número 129. pero del año de 1858.

135 Anverso.- Sello ochavado, con el monograma de las letras L A N C R I (Plancarte) (14).

Reverso.-Liso.

Cobre. Eliptica. $20 \mathrm{~mm}$. por $9 \mathrm{~mm}$.

Hay varias otras formas y tamaños.

136 Anoerso.-Monograma délas letras P L A N C A R T E S.

Reverso.- - Liso.

Cobre. $25 \mathrm{~mm}$.

137 Anverso.-Aguila mexicana. Abajo, guirnalda foliácea. Leyenda: J. SIERRA.

Reverso.-Liso.

Cobre. $20 \mathrm{~mm}$.

Moneda utbana mercantil.

138 Anverso.-El monograma de las letras $J$ A $O$ E, rodeado de una orla, en forma de cadena por una parte y foliaicea por la otra.

Reverso.-Liso.

Cobre. $18 \mathrm{~mm}$.

(14) La familia P'ancarte era una de las más prominentes de Zamora. El Obispo dé Cuernavaca y más tarde Arzobispo de Linates. D. Francisco Plancarte y Navarrete, eminente atqueólogo e historiador, reunió una importante colección numismática, en la que figuraba buen numero de las monedas de necesidad de Michoacán. 
139 Anverso.-El monograma de las letras F M R P A, incuso. Encima, un adorno. Reverso.-Liso.

Cobre. $22 \mathrm{~mm}$.

140 Anverso.-Sello octágono; dentro de dos círculos concéntricos, el exterior foliáceo. la leyenda: ESQUINA DE ZEBALLOS.

En el centro, $Z A$.

Reverso.--Liso.

Cobre. $27 \mathrm{~mm}$.

Moneda urbana mercantil.

141 Anverso.-Dentro de dos círculos concéntricos, el exterior de adorno y el interior foliáceo, la leyenda: PEDRO RODRIGUEZ FERNANDEZ. Sobresellada con $Z$ A en el centro.

Reverso.-Liso.

Cobre. $26 \mathrm{~mm}$.

Moneda urbana mercantil.

142 La moneda número 130, de Zamora, sellada al reverso con $Z_{A}$ dentro de un pequeño círculo, con gráfilas de pequeñas rayas.

143. La número 134, con dicho sello.

144 Moneda igual a la número 123, sellada al reverso con las letras $\mathrm{ZA}$. dentro de un círculo.

145 Como la anterior, pero el resello ZA. se encuentra dentro de un marco cuadrado de pequeños picos.

146 Moneda de Ixtlán, resellada al anverso con $\mathrm{ZA}_{\mathrm{A}}$ dentro de un circulo.

147 La moneda número 126, resellada al anverso con la fracción $1 / 8$ dentro de un pequeño círculo.

148 Arbitrio de Penjamillo, resellado al anverso con la fracción $1 / 8$ dentro de un pequeño cítculo.

$149 \mathrm{El}$ mismo resello en la moneda número 123. Algunos dob!es.

150 La moneda número 129 , sellada al reverso con $\mathrm{ZA}$. y la fracción $1 / 8$, dentro de un círculo cada una.

151 Moneda lisa, resellada como la anterior.

152 La moneda número 123, contramarcada al anverso con una letra $M$.

153 Anverso.-La inscripción en dos lineas: MOD-MAIS (medio de maíz). Gráfila en forma de cadena.

Reverso.-Liso.

Cobre. $20 \mathrm{~mm}$.

Tanteo de maiz del Distrito de Zamora. 
154 Anverso.- Monograma de las letras M $\AA 1 \mathrm{~s}$.

Reverso.-Liso.

Cobre. Octágono. 22 por $21 \mathrm{~mm}$.

Tanteo de maiz de Zamora.

155 Anverso.-Monograma de las letras MO D M S (Medio de maiz).

Reverso,-Liso.

Cobre. Pentágono. $19 \mathrm{~mm}$. por $14 \mathrm{~mm}$.

Tanteo de maiz de Zamora.

156 Anverso.-Sello cruciforme de las letras O - E V S E - D M A S (Eusebio? De maiz).

Reverso.-Liso.

Cobre. $20 \mathrm{~mm}$.

Tanteo de maiz de Zamora.

\section{DUDOSAS}

1 En el anverso de una cuartilla de Jalisco, las letras Te Po (Topete-Penjamillo?). Un sobresello con las letras $T, E$, cursivas, dentro de una guirnalda foliácea. Al reverso, un sello con la inscripción en cursivas. .. . de Topete.

2 Como la anterior, más, al anverso, el sello MA. de Morelia.

3 Tlaco de la Federación, con el sobresello T. E., como en el número 1.

4 Dentro de un cítculo de adorno, las letras GG, entre rosetas. En el exergo, PILON. Reverso liso. Cobre, $13 \mathrm{~mm}$. (Hacienda del Pilón?)

5 Dentro de un octágono de picos menudos la inscripción en dos lineas STA.EFYGa. Abajo un adorno, Reverso liso. Cobre $25 \mathrm{~mm}$. (Hacienda de Santa Efigenia?).

\section{OBRAS CONSULTADAS}

Catálogo de la Colección Fischer. Nueva York, 1891.

Catálogo de ta Colección Guttag. Nueva York, 1929.

Antonio Gatcia Cubas.-Diccionario Geográfico, Histórico y Biográfico de los Estados Unidos Mexicanos, México, 1888-1891.

Dr. Nicolós León.-Las Monedas de vidrio de Oaxaca. En "Conozca Usted a México", México, 1924.

Dr. Nicolás León.-Numismátiça Quiroguense. En "El Estandarte". San Luis Potosi, 1899.

Manuel Orozico y Berra.-Acuñacióı en México. En "El Renacimiento", México, 1869.

José Guadalupe Romero.-Noticias para formar la historia y la estadistica del Obispado de Michoacán. México, 1862.

Manuel Romero de Terreros.-Los tlacos coloniales. México, 1935.

$F$ von Schrötter.-Mexikanische Haciendamarken. Berlin, 1932.

Adolf Weyl.-Catálogo de la Colección Fontobert. Berlín, 1878. 